Received: 7 December 2012, Accepted: 19 June 2013

Edited by: J. J. Niemela

Reviewed by: V. Lakshminarayanan, Waterloo University, Canada

Licence: Creative Commons Attribution 3.0

DOI: http://dx.doi.org/10.4279/PIP.050005

www.papersinphysics.org

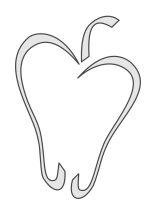

ISSN 1852-4249

\title{
Enhancement of photoacoustic detection of inhomogeneities in polymers
}

\author{
P. Grondona, ${ }^{1}$ H. O. Di Rocco, ${ }^{2}$ D. I. Iriarte, ${ }^{2}$ J. A. Pomarico, ${ }^{2}$ \\ H. F. Ranea-Sandoval, ${ }^{2 *}$ G. M. Bilmes ${ }^{3}$
}

\begin{abstract}
We report a series of experiments on laser pulsed photoacoustic excitation in turbid polymer samples addressed to evaluate the sound speed in the samples and the presence of inhomogeneities in the bulk. We describe a system which allows the direct measurement of the speed of the detected waves by engraving the surface of the piece under study with a fiduciary pattern of black lines. We also describe how this pattern helps to enhance the sensitivity for the detection of an inhomogeneity in the bulk. These two facts are useful for studies in soft matter systems including, perhaps, biological samples. We have performed an experimental analysis on Grilon ${ }^{\circledR}$ samples in different situations and we show the limitations of the method.
\end{abstract}

\section{Introduction}

In highly light-scattering materials, such as certain types of polymers, turbid liquids, glassy structures, and body organs, inspection and monitoring of internal features were made possible by means of $\mathrm{X}$ Ray irradiation until the development of ultrasound imaging. The former has the well-known disadvantage that in biological tissues it may trigger degenerative processes in the cells, and in non-biological samples, X-Ray inspection is not always simple to perform directly in the production line. Ultrasound imaging is very helpful in these situations.

On the other hand, visible light optical tomogra-

*E-mail: hranea@exa.unicen.edu.ar

1 Universidad Nacional de Rosario. Facultad de Ciencias Bioquímicas y Farmacéuticas. Rosario (Santa Fe) Argentina.

2 Instituto de Física "Arroyo Seco", Universidad Nacional del Centro de la Provincia de Buenos Aires. Calle Pinto 399, B7000GHG, Tandil (Buenos Aires) Argentina.

3 Centro de Investigaciones Ópticas (CONICET-CIC) and Facultad de Ingeniería Universidad Nacional de La Plata, La Plata. Argentina. phy and optical topography is nowadays reaching the status of clinical resource in the detection and monitoring of several types of tumors and for noninvasive evaluation of oxygenation of tissues in biological samples. In non-clinical applications it can be used for the detection of abnormal bodies within materials, which is of great importance in quality control in several areas of technology. These techniques were derived from the study of light propagating in turbid media, and applied afterward to biological samples and medical imaging of different parameters often using polymers as phantoms of biological tissues [1-7].

The photoacustic effect (PA) provides a method of analysis that has been used in clear fluids and has sufficiently proven its capability for detecting very low concentrations of absorbing species in a mixture or solution; it has also been used for the monitoring of molecular processes in different environments as shown in references [7-17]. This paper intends to make a contribution on the application of the PA in soft matter, namely the detection of inclusions in polymer samples and the direct determination of the speed of sound in the material used 
Papers in Physics, vol. 5, ART. 050005 (2013) / P. Grondona et al.

for the samples.

The PA technique has the advantage that acoustic waves do not scatter as light does in the characteristic lengths of many experimental situations. Even if the excitation light undergoes scattering, the location of an inhomogeneity within the bulk of the sample can be achieved by detecting the remnant of the shock wave generated at an absorbing region or at an interface at which the speed of the sound waves changes. Repeating this inspection at other relative positions of the laser and the acoustic detector and with the aid of a suitable algorithm, a sufficiently precise location of a single inhomogeneity of simple geometry can thus, in principle, be resolved, together with some information about its composition (using at least two wavelength for the excitation), provided the speed of sound is known (see, for example, Ref. [20]). An example of this is presented in Ref. [21] in a rear-detection scheme used for detecting inhomogeneities in subsurface inhomogeneities in metals.

The PA detection of bodies included in a turbid medium may provide complementary information to diffuse light propagation studies in that medium. Namely, it could bring an independent value for the absorption coefficient, and it thus may help in the solution of the inverse problem in optical tomography of samples.

The speed of sound determination relies on the fact that the acoustic signal picked by the transducer arrives at times proportional to the distance from the laser beam that generates the shock wave to that transducer. A drawback with the photoacustic method applied to turbid materials is that the light scattered by the bulk generates a pressure pulse on the detector if it is in contact with a free surface of the sample explored. Consequently, the time of arrival of the pressure signal at the detector is insensitive to the relative position of the laser and the sensor. Hence, the speed of the waves involved in the PA signal is difficult to determine and requires an adequate procedure to evaluate it. This is one of the motivations of this contribution.

For this paper, we used a laser pulsed photoacustic system equipped with a PZT in contact with the sample made of polymer Grilon ${ }^{\circledR}$ which is representative of a turbid medium, to show how the presence of controlled, fiduciary absorbing regions in the surface of a sample are used as local wave generators that allow the determination of the speed of waves in materials despite of the light scattering described. We have engraved in the surface of the samples a pattern of stripes of absorbing material. In this way, we have a greater signal whose contribution may be discriminated from the signal generated by the light scattered by the sample material. We demonstrate that this fiduciary pattern is useful also to enhance the photoacustic signal, and that from that signal the presence of inhomogeneities in a medium may be inferred.

Other successful recent approaches to the problem of detection of tumoral tissues in biological samples can be found in Refs. [18,19].

\section{Experimental}

A scheme of the pulsed photoacustic system used in all the experiments is shown in Fig. 1, which is essentially the same that can be used to determine the speed of sound in liquids and in clear samples.

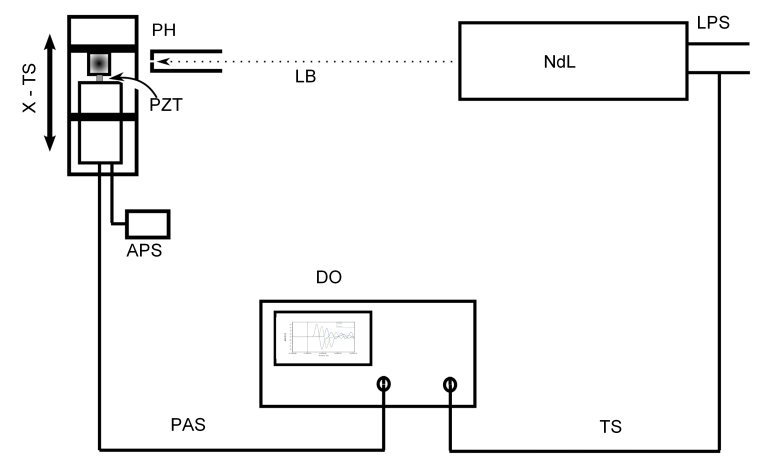

Figure 1: Experimental setup of the experiment. The parts are: the $\mathrm{Nd}^{+3}$ :YAG laser $(\mathrm{NdL})$, the positioning device (X-TS), the Pinhole $(\mathrm{PH})$ to clip the laser beam (LB), and the oscilloscope (DO), the amplifier with a DC power supply (APS). The DO is synchronized with the laser via the laser pulse synchronization (LPS) cable.

We used a pulsed $\mathrm{Nd}^{+3}$ :YAG laser emitting at $1.06 \mu \mathrm{m}$ with a pulse duration of approximately 10 $\mathrm{ns}$, at energies between $0.5 \mathrm{~mJ}$ to $50 \mathrm{~mJ}$. The laser beam was clipped by means of a pinhole in order to reduce the original laser beam size and to use 
a uniform spot thus reducing the power impinging on the samples. This pinhole was held at the far end of a beam dump for security reasons. In the results we present here, we have used two pinholes of $1 \mathrm{~mm}$ and $1.5 \mathrm{~mm}$ in diameter which shall be specified in each experiment. This is the diameter of the laser impinging on the sample, as inferred from sensitive photographic paper. For acoustic detection, a ceramic $4 \times 4 \mathrm{~mm}^{2}$ PZT transducer was strongly pressed against one of the free surfaces of the sample, namely the one normal to that facing the laser. The photoacustic signals were amplified and processed by means of a Tektronix TDS 3032B, $300 \mathrm{MHz}$ digital oscilloscope, averaging at least 64 signals before displaying the photoacustic signal.

Samples used were square-section parallelepipeds, $10 \mathrm{~mm}$ width, and $39 \mathrm{~mm}$ high, all made from the same polymer Grilon ${ }^{\circledR}$ piece. The samples were placed in a C-clamp, with the PZT cage in one of its arms. The fiduciary pattern engraved on one of the faces of some samples consists of five grooves of approximately $1 \mathrm{~mm}$ width and $0.2 \mathrm{~mm}$ deep, filled with thick black paint, separated by stripes of material which retain the natural turbid white color of the polymer (which we call "clear" for short) of $1 \mathrm{~mm}$, whose lengths are approximately $70 \%$ the length of the face. A second type of sample prepared in a similar fashion, but with a centered cylindrical hole of 3 $\mathrm{mm}$ diameter drilled in it parallel to the surfaces of the sample in all cases mentioned, was also used in the experiments in order to compare the signals with the former. This cavity was alternatively emptied or filled with deionized water. We call "sample 1" the one drilled with the cylindrical cavity, and "sample 2" the one without the hole. Figure 2 is a sketch of sample 1 with a schematic representation of the fiduciary pattern used. The PZT and the laser beam relative positions are displayed, together with the approximate position of the cavity.

We obtained two types of signals, those from samples without holes and those from samples with centered holes. Each type was subdivided into signals taken with the laser impinging on the blank surface, and those taken with the laser impinging on the patterned surface. Besides, there are sig-

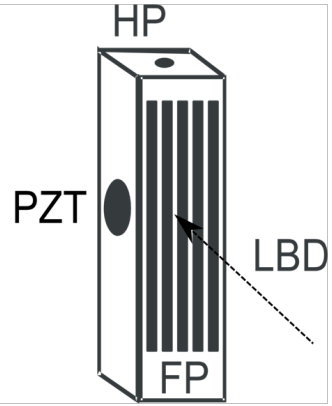

Figure 2: The Grilon ${ }^{\circledR}$ Sample prepared for the surface absorption experiments. The shadowed region (PZT) is the location of the transducer with respect to the impinging laser beam direction (LBD). The cavity is a $3 \mathrm{~mm}$ diameter hole, whose position (HP) is shown for the samples that have drilled cavities. The cavity may be empty or filled with water. The height of the samples is $39 \mathrm{~mm}$ and has a 10 $\mathrm{mm}$ square base. It has five grooves (FP) in its front face, painted in black to enhance absorption.

nals obtained from the samples with cavities, either empty or filled with water.

In each sample, the laser point of impact was moved from the farthest possible position to the nearest with respect to the PZT. This was accomplished by means of a $1 \mu \mathrm{m}$ precision, step motor movable stage, Zaber Model T-LA60A, controlled by a PC interface.

\section{Results}

In order to properly analyze the results, we calibrate the response of the system to increasing laser pulse energy. To this end we irradiate a blank surface of sample 2 at a point near the center of the face, and we plotted the amplitude of the first maximum of the acoustic signal as a function of the laser pulse energy. The result is displayed in Fig. 3 and shows linearity in the energy range used.

In the same plot, we display three points (including the origin) which are the maxima of the signal at the same location of a striped sample face, but impinging on a black groove. As it can be seen, the signal nearly trebles its maximum peak for the same excitation energy. In both experiments, the pinhole used was $1.5 \mathrm{~mm}$ in diameter. 


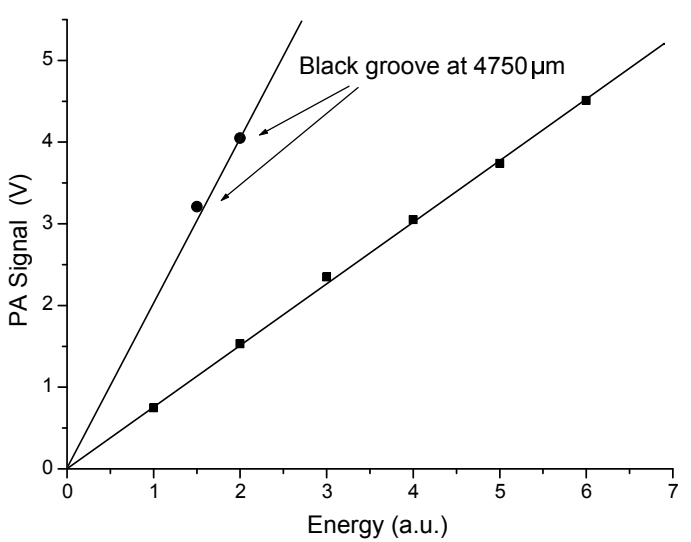

Figure 3: PAS vs. laser pulse energy. The linearity of the PA response (squares) to laser excitation is evident in the plots. The black dots represent the PA signal when the laser impinges on a black stripe nearly at the center of the front face of a striped sample.

After ascertaining the linearity of the response, and the fact that there is an evident dependence of the PAS on the absorbance of the surface, we obtain a profile of three of the grooves of sample 2 by plotting the value of the amplitude of the first peak of the PA signal versus the relative distance between the PZT and the excited region using the same pinhole as before. The result of this is shown in Fig. 4. It can be seen that 1) the groove profile is neatly resolved, and 2) there is an improvement of the signal generated in the black stripes which decreases as the distance increases. Since the stripes and the laser beam have approximately the same transverse size of the grooves, the resulting profile is somehow rounded off, but this is not important in what we aim to prove here.

We could determine the speed of sound in the sample from a plot of the time position of the beginning of the first peak of the acoustic signal (arrival time), as a function of the distance between the impinging point on the sample and the PZT detector. But when we try to do that, experiments demonstrate that the time elapsed since a laser pulse triggers a digital oscilloscope and the appearance of the PA signal is the same regardless of the distance between the impinging laser beam and the detector,

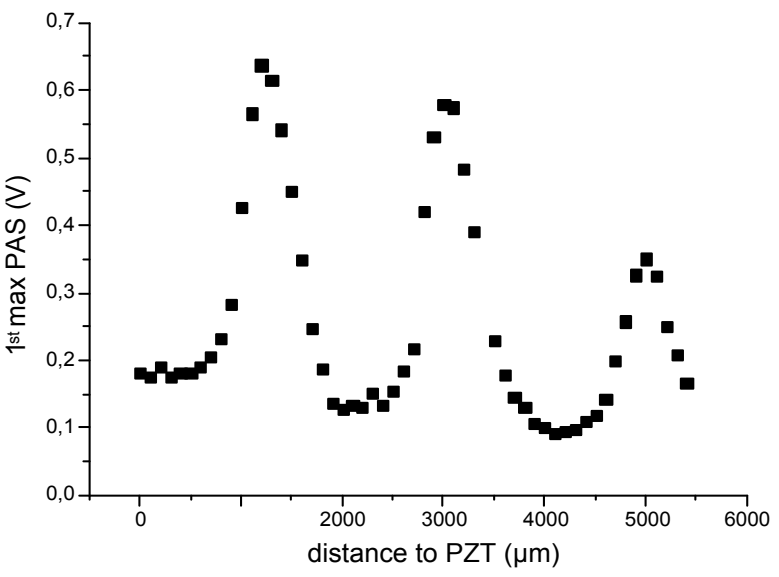

Figure 4: The centers of clear bands and black grooves are clearly resolved by scanning the surface with the laser. The signals were taken at $100 \mu \mathrm{m}$ displacement from each other. The energy of PAS decreases with distance of the laser beam to PZT. The increment in signal due to the grooves is more than 6 -fold with respect to the signal due to the bulk polymer.

due mainly to the light scattered by the bulk of the polymer that hits the PZT. This poses a problem in the evaluation of the speed of the waves.

To avoid that difficulty, we use the signal produced if the laser hits in the black grooves, generated only by the absorption at the grooves. We obtain this by subtracting from the PAS signal measured when the laser impinges in a black groove, the PAS signal obtained in a "clear" region nearby. To this end, we moved the sample slightly away from the previous black stripe, so the first PAS signal was obtained with the beam impinging in a black groove and the second PAS signal was obtained with the beam impinging in a white stripe.

Figure 5 shows the determination of the speed of sound in sample 2 by this method. Since the plot uses as input the maxima of the amplitude of the signals, the straight line would not cross the origin. The extrapolated value for zero-crossing corresponds approximately to the amplitude of the first maximum of the signal in clear samples.

Evaluation of the slope of the resulting line allows the calculation of the value of speed, as $v=$ $(2333 \pm 133) \mathrm{ms}^{-1}$, which compares well with calculated data determined by using the properties of 


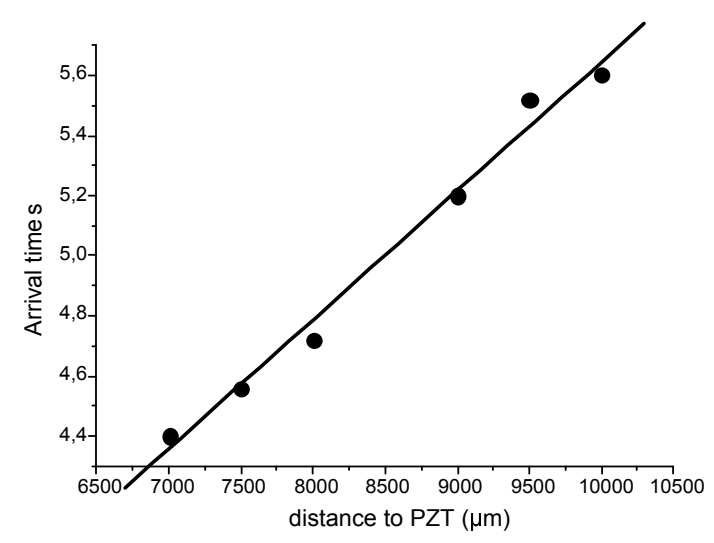

Figure 5: Time of the first maximum of the processed signals vs. the position of the impinging point of the laser presents a linear correlation $(R \approx 0.994)$ that yields a value of $v=(2333 \pm 133)$ $\mathrm{ms}^{-1}$ for the speed of sound. All these signals were taken with a pinhole of $1 \mathrm{~mm}$ diameter.

the polymer [22]. Since the vibration of the whole sample has distinctive frequencies, the FFT of the PA signal provides another estimation of the speed of waves, once the frequency sequence is properly found. The Fast Fourier Transform (FFT) analysis was used both, to estimate the sound speed in the Grilon ${ }^{\circledR}$ sample via the frequencies identified in the spectrum and their spacing, knowing that the piece is a parallelepiped of known dimensions, and to define a scale for the energy of the pulse. The details of this procedure are straightforward calculations [23]. We found it useful to use the power spectrum for defining the energy instead of the integral of the temporal pulse and that is the parameter we use in the presentation of the results.

Figures 6 and 7 show an FFT treatment of the signals obtained from the following three cases: solid Grilon ${ }^{\circledR}$ sample, sample with empty cavity and sample with the hole filled with deionized water. The PAS energy used in this figure is a measure of the energy content of the acoustic pulse, as evaluated from the power spectrum of the signal.

In Fig. 6, we display the results obtained impinging with the laser on clear faces, and in Fig. 7 we show the results impinging with the laser on the patterned faces. It is clear that a distinctive feature arises near the center of the sample in the patterned faces where a black stripe is located, which is not visible in the clear-face analysis.

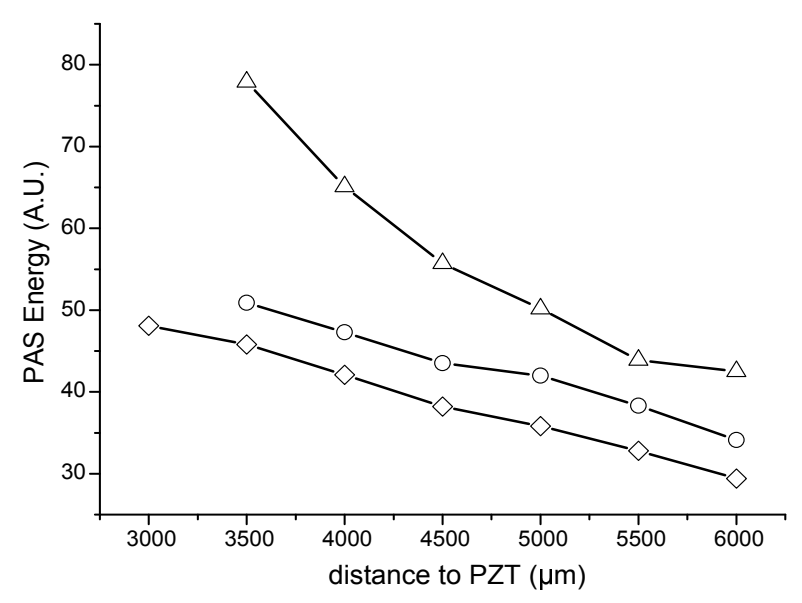

Figure 6: The acoustic energy (FFT power integral of the PAS) vs. the relative distance between the laser beam and the PZT in Grilon ${ }^{\circledR}$ samples in a face with no fiduciary pattern (clear sample). The energy diminishes as the distance to the detector increases. References in the insert: Triangles represent the cavity empty in a clear sample. Circles are for cavity filled with water in clear samples. Rhombi are for clear samples without the cavity.

The acoustic energy deposited in the patterned faces is more than one order of magnitude higher than that obtained in the clear faces when the inclusion is present (compare Fig. 6 with Fig. 7). The water-filled hole and the empty hole are also clearly distinguishable from the solid Grilon ${ }^{\circledR}$ response.

\section{Analysis and Conclusions}

We have performed an experimental analysis of the photoacustic signal in Grilon ${ }^{\circledR}$ polymer but it can be extended to other materials as epoxy resins used also as biological phantoms. We have shown that such applications are viable for quantitative determinations. The above results can be used to determine the presence and some optical characteristics of an inhomogeneity embedded in this type of materials.

All the acoustic signals detected by the PZT in this soft turbid solid material begin at approximately the same time after the laser trigger fires, regardless of the relative distance from the impact 


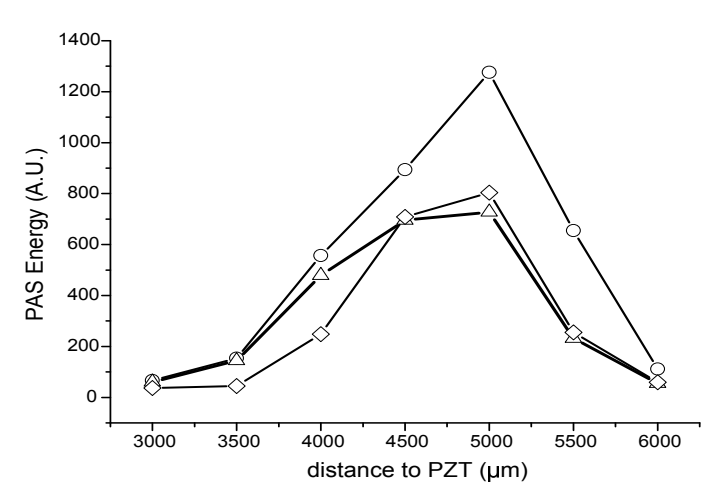

Figure 7: The energy deposited by the laser on a white stripe and on the black grooves for the three cases analyzed as seen in the insert. The circles represent a grooved sample with the cavity filled with water. Triangles and rhombi are for grooved samples and the empty cavity, and no cavity, respectively.

point of the excitation to the PZT acoustic detector, due mainly to scattering, making this method useless to evaluate the speed of sound by the scanning standard procedure.

The differences in those PA signals are difficult to analyze. Therefore, to evaluate the speed of the acoustic waves and to gather information about the presence of a cavity or inhomogeneity in the polymer, we have developed a method that used a regular pattern on the surface of the sample, consisting on parallel clear stripes of the base material and grooves filled with highly absorbent black paint. In the black grooves, the localized absorption provides a strong shock wave at the surface. By comparing the time of appearance of the signal in different positions of the surface, it is possible to estimate the speed of those waves in the polymer.

For each of the zones in the pattern, the PA curves undergo a change of shape and amplitude from signals in the white zones to the signals obtained in the black stripes, being this strong evidence of the effect of the inhomogeneity in the signal. A Power FFT was performed on each signal in order to provide another means to determine whether a black or a white stripe is excited, and the integral of the FFT provides a measure of the energy absorbed by the sample in each case. Please note that the energy of the laser was fixed to a value that avoids bleaching of the paint, being in all cases below $500 \mu \mathrm{J}$ per pulse.

The signals generated at these inhomogeneities provide a well defined point of absorption and thus a definite path for the sound generated by the light-absorption mechanism which is very distinctive from other mechanisms of excitation of the PZT. It also reveals the presence of a surface inhomogeneity once the contributions of other sources of acoustic waves are identified making suitable use of reference signals. All the conclusions of this work must be under the proviso that the PZT has a limited frequency band.

Although the above results were obtained for a soft polymer with a fiduciary painted pattern, they can be extended to other type of resins with charge of dyes or other absorbent particles. We are confident that with minor modifications it can be used for the determination of properties of materials of biological interest as well.

The results shown in Figs. 6 and 7 confirm that employing one of the surfaces of the sample conveniently patterned, and scanning it for detection of ultrasound signals, can be used to determine the presence of an inhomogeneity, albeit its precise location and size is not well defined by this procedure and it should be complemented by similar determinations at other relative positions of the laser and the PZT. The increase in the signal with respect to the background material is at least one order of magnitude or better.

When using this technique in phantoms used in medical applications, one should take care of the fact that there are limitations in several aspects, such as the power involved in each pulse avoiding any kind of damage, and that using other wavelengths would be better suited for biological tissues which involve blood. Other type of samples are being currently inspected by modifications of the procedure reported here so to adapt it to gelled phantoms.

The conclusions are, in short, that the system is sensitive to the presence of the inhomogeneity, and that the higher absorbance of the painted stripes in the surface allows not only to evaluate the speed of sound (which is essential to any tomographic technique) but also improves the detectivity by enhancing the energy released as mechanical waves. This 
Papers in Physics, vol. 5, ART. 050005 (2013) / P. Grondona et al.

is a non-trivial result since in the modeling of the propagation of the laser light in the turbid substance, scattering is predominant, but still is sufficient for the detection of inhomogeneities through changes in the absorption. The technique based on the PA is simple and has the advantage that it can be adapted to be used in larger samples or in samples of biological interest. The procedure of using a single acoustic detector for the signals produced by the laser scanning of the surface under study, has an advantage over multiple detector arrangements in the sense that with a suitable fiduciary pattern the method can provide information about the speed of the waves involved in the signal. This is interesting because the data processing would not depend on generic information about its value.

Acknowledgements - PG wants to thank the Red Nacional de Laboratorios de Óptica for financial help and partial funding during the experiments and to InterU System for providing a grant for the completion of the experiments. This work partially funded by Universidad Nacional del Centro de la Provincia de Buenos Aires, Agencia Nacional de Promoción Científica y Tecnológica (PICT 0570) and CONICET (PIP 384). HODR, DII, JAP and HFRS are members of Carrera del Investigador Científico, Consejo Nacional de Investigaciones Científicas y Técnicas (Argentina). GMB is member of Carrera del Investigador Científico, Comisión de Investigaciones Científicas de la Provincia de Buenos Aires (Argentina). Authors wish to thank Nicolás A. Carbone for help in the final preparation of the manuscript.

[1] A Ishimaru, Wave propagation and scattering in random media, Academic Press, New York (1978).

[2] J Ripoll Lorenzo, Light diffusion in turbid media with biomedical applications, $\mathrm{Ph}$. D. Thesis, Universidad Autónoma de Madrid, Spain (2000).

[3] A K Dunn, H Bolay, M A Moskowitz, D A Boas, Dynamic imaging of cerebral blood flow using laser speckle, J. Cerebr. Blood F. Met. 21, 195 (2001).
[4] P N den Outer, Th M Nieuwenhuizen, Ad Lagendijk, Location of objects in multiplescattering media, J. Opt. Soc. Am. A 10, 1209 (1993).

[5] D A Boas, M A O'Leary, B Chance, A G Yodh, Detection and characterization of optical inhomogeneities with diffuse photon density waves: a signal to noise analysis, Appl. Opt. 36, 75 (1997).

[6] D Contini, H Liszka, A Sassaroli, G Zaccanti, Imaging of highly turbid media by absorption method, Appl. Opt. 35, 2315 (1996).

[7] A C Tam, Applications of photoacustic sensing techniques, Rev. Mod. Phys. 58, 381 (1986).

[8] C K N Patel, A C Tam, Pulsed optoacoustic spectroscopy of condensed matter, Rev. Mod. Phys. 53, 517 (1981).

[9] A A Oraevsky, A A Karabutov, Optoacoustic tomography in Biomedical Photonics, Ed. Tuan Vo-Dinh, CRC Press, Chapter 17 (2002).

[10] R O Esenaliev, A A Karabutov, A A Oraevsky, IEEE J. Sel. Topics in Quantum. Electron. 5, 981 (1999).

[11] L Nicolaides, A Mandelis, M Munidasa, Experimental and image-inversion optimization aspects of thermal wave diffraction tomography microscopy, AIP Conf. Proc. 463, 8 (1998).

[12] P C Beard, Photoacustic imaging of blood vessel equivalent phantoms, Proc. SPIE 4618, 54 (2002).

[13] E Zhang, J Laufer, P Beard, Backward-mode multiwavelength photoacustic scanner using a planar Fabry-Perot polymer film ultrasound sensor for high-resolution three-dimensional imaging of biological tissues, Appl. Opt. 47, 561 (2008).

[14] S Fantini, M A Franceschini, E Gratton, Quantitative determination of the absorption spectra of chromophores in strongly scattered media. A light-emitting-diode based technique, Appl. Opt. 33, 5204 (1994). 
[15] G M Bilmes, O E Martínez, P Seré, D J Orzi, A Pignotti, On line photoacustic measurement of residual dirt on steel plates, AIP Conf. Proc. 557, 1944 (2001).

[16] K H Song, E W Stein, J A Margenthaler, L V Wang, Noninvasive photoacoustic identification of sentinel lymph nodes containing methylene blue in vivo in a rat model, J. Biomed. Opt. 13, 054033 (2008).

[17] Z Xu, Ch Li, L V Wang, Photoacustic tomography of water in phantoms and tissue, J. Biomed. Opt. 15, 036019 (2010).

[18] L Xi, X Li, L Yao, Design and evaluation of a hybrid photoacoustic tomography and diffuse optical tomography system for breast cancer detection, Med. Phys. 39, 2584 (2012).

[19] B Wang, Q Zhao, Photoacoustic tomography and fluorescence molecular tomography: A comparative study based on indocyanine green, Med. Phys. 39, 2512 (2012).
[20] M Xu, L V Wang, Photoacoustic imaging in biomedicine, Rev. Sci. Instrum. 77, 041101 (2006).

[21] R Takaue, H Tobimatsu, M Matsunaga, K Hosokawa, Detection of surface grooves and subsurface inhomogeneities in metals by transmission correlation photoacoustics, J. Appl. Phys. 59, 3975 (1986).

[22] Data on mechanical properties of Grilon can be found in http://engr.bd.psu.edu/rxm61/METBD470/ Lectures/PolymerProperties\%20from\%20 CES.pdf and in http://www.inoxidable.com/ propiedades1.htm.

[23] P Grondona, Caracterización de un sistema fotoacústico en el IR cercano para estudios en medios turbios. Algunas aplicaciones al estudio en fantomas de polimeros con inclusiones, Master Degree Thesis, Universidad Nacional de Rosario, Argentina (2009). 